disciplinary (MDT) approach at Kirkwood, with input offered by our trained Counsellors and Specialist Palliative Care Social Workers. Working closely with MDT members, we hope to develop this group, along with the service users, so that it is truly meeting the needs identified by the group.

Feedback from Kirkwood's service and carer user forum had previously indicated a demand for a male-specific service at Kirkwood Hospice. This was alongside recognition of existing services offered perhaps not appealing to male patients. Given the national 'Men's Shed' movement has been gaining momentum and demonstrating positive links with mental health and wellbeing, it has felt like the right opportunity to develop a Men's Space session.

With the aim to engage and support more male service users at Kirkwood, the 'Men's Space' sessions have been developed and launched. The sessions are being envisaged as a developmental 12 week programme where service users, alongside Kirkwood Hospice staff, come together in a supportive manner with the option to plan and deliver a specific project such as gardening, creative activities or IT based projects. The activities undertaken by the group will be led by the group itself and supported and facilitated by staff from the Support and Therapy team, Family Care Team and IT team members. Users can attend for as long as they want to during a weekly four hour period.

Initial feedback has been extremely positive and the service will be evaluated through the 'Short Warwick-Edinburgh Wellbeing Scale' at week 1, 6 and 12. The sessions are also being promoted to local stakeholders including other Men's Sheds groups based in Kirklees.

\section{P-26 CREATING A TEMPLATE TO IMPROVE RECORDING END OF LIFE DISCUSSIONS/PLANS IN A CUSTODIAL SETTING}

${ }^{1}$ Leone Beet, ${ }^{1}$ Tracy Rhodes, ${ }^{2}$ Jill Bennett. ${ }^{1}$ St Catherine's Hospice, Preston, UK; ${ }^{2}$ Wymott Prison, Leyland, UK

\subsection{6/bmjspcare-2019-HUKNC.50}

Background In April 2018 the Dying Well in Custody Charter was published. This Charter provides a framework to assist all prison staff supporting an individual who is preparing to die in a custodial setting. Projections indicate the number of individuals aged 50 plus held in custodial settings is increasing rapidly. There is strong evidence that this older population in prisons experience higher burden of physical/mental health problems. Prison staff highlight that initiating discussions about end of life care/preferences in relation to place of death/care was challenging and that the recording and tracking of these discussions/plans was problematic leading to possible unnecessary hospital admissions.

Aims Design/develop an end of life template focused on the Dying Well in Custody Charter. The template to be given a designated area on the computer system (SystmOne) that all prisons use nationally. The template can be used as a passport of end of life discussions/planning if the individual moves to another location e.g. other prison, hospice, community setting. Provide multi-professional education focused on planning/ recording of end of life care in prisons.

Method Collaborative working with hospice and two prison healthcare/IT teams. Staff comments/ideas gained through group discussions to develop the template.
Implementation To trial the template in two prisons and one hospice over six months. First prison has a high percentage of elderly individuals and the second prison has a health-care unit. The hospice uses the same computer system and covers both prisons.

Evaluation Discussion of the use of the template monthly and amend as appropriate until a template has been formed that meets the need of individuals and can be used nationally to improve end of life care/planning in custody.

\section{P-27 INNOVATIVE PARTNERSHIP WORKING TO HELP SUPPORT THE NEEDS OF DIVERSE COMMUNITIES}

Susan Smith, Kathryn Hamling. Highland Hospice, Inverness, Highlands

\subsection{6/bmjspcare-2019-HUKNC.51}

Highland Hospice covers an area 20\% larger than Wales with a population less than $8 \%$ the size. The delivery of services equitably has proven extremely challenging. Whilst the concept of a hospice running a volunteer befriending service is not innovative, we believe our approach to rolling this out across the Highlands through partnership with local communities is.

Helping Hands is a volunteer befriending and support service established in 2016. The service has four objectives: build community capacity to deal with death and dying; support carers to sustain their caring roles; mitigate impacts of social isolation for clients; support wider health and social care system to cope with increasing demand.

The service is open to all individuals in need, regardless of their diagnosis. In order to extend the service across the whole Highland region we deliberately sought small, locally run organisations with a close connection to their community. To date we have established five partnerships each at a different stage of development. One partner had an existing befriending service we were able to augment, the others had no similar services. The partnerships work with small populations dispersed across large areas. Local knowledge and contacts support the recruitment of volunteers and helps people feel more comfortable referring to the service.

Seeing success, the hospice has committed to establishing more partnerships to develop local services. Our 2018-2021 strategy, 'Supporting our Compassionate Community', reflects the fact that we wish to support our wider community so that together we can help address palliative and end of life care needs.

We recently identified two rural Highland communities with under-provision of social care who want to work with us to pilot partnership delivery of social care services. Working in this new way can prove challenging but the opportunities of supporting people in their own community is worth it.

\section{P-28 SAFEHARBOUR: SUCCESSES AND CHALLENGES IN THREE YEARS OF OUTREACH TO UNDER-SERVED GROUPS IN NORTH EAST ESSEX}

Debbie Pigeon, Karen Chumbley, Lee Welland. St Helena, Colchester, UK

\subsection{6/bmjspcare-2019-HUKNC.52}

Background The Safe Harbour project was set up in 2016 to address the lack of hospice support for patients from underserved groups. During its pilot phase, a feasibility study 
established that there was poor access to hospice support for vulnerable people.

Aims To enable vulnerable people in North East Essex to live well with their life limiting illness and die with dignity in a place of their choice.

Methods A three-year project, funded by Macmillan was set up to facilitate the services needed to enable the aims to be reached. The focus was on those from the local homeless community, those living in areas of deprivation and those identified through the lung cancer early diagnosis scheme. As referrals increase from these groups, the future aim is to focus on networking with other support groups and improve hospice care for patients with mental illness, learning disabilities, LGBTQ+ groups and refugees.

Results

1. Successful liaisons with homeless support groups, neighbourhood schemes and services for vulnerable people in North East Essex;

2. Joint working between lung oncology services and hospice to improve the uptake of palliative support for patients with lung cancer;

3. A Macmillan information hub, staffed by volunteers, providing information to patients;

4. Referrals have increased by $51 \%$ in areas of deprivation due to improved communication/liaison with local support groups;

5. Patients have included homeless, drug and alcohol users, hoarders, patients with learning disabilities, mental health issues and those from ethnic minority groups.

Conclusion By providing information about the hospice services to the support groups and neighbourhood schemes local knowledge has improved. The service and referrals to the hospice for the most marginalised of our population have grown.

\section{P-29 COMMUNITY REPRESENTATION AND CULTURAL DIVERSITY OF THE MODERN-DAY HOSPICE}

Anne-Marie Bourke, Maxwell Charles. Marie Curie Hospice, Newcastle upon Tyne, UK

\subsection{6/bmjspcare-2019-HUKNC.53}

Background National guidance advocates that patients living with life limiting illnesses have access to individualised palliative care (General Medical Council, 2010). Evidence suggests that Black and Asian Minority Ethnic (BAME) groups are less likely than White patients to access palliative services (Markham, Islam \& Faull, 2014; Tackling Inequalities in End of Life Care for Minority Groups VCSE Health and Wellbeing Alliance Project Group, 2018). Negative consequences of this include patients experiencing symptoms and being denied the opportunity to consider advance care planning.

Aims The aim of this service evaluation was to establish the extent to which the documented ethnicity of patients referred to our hospice inpatient (IPU) and day therapies units (DTU) compare to the ethnic diversity of our local community (taken from the 2011 electoral ward census data).

Methods Documented ethnicity data of all patients admitted to the IPU or accepted onto the DTU caseload between November 2017 and December 2018 were retrospectively collected from SystmOne. Census data (2011) for the hospice electoral ward were obtained from the Office for National Statistics.
Results The populations of our IPU and DTU were not representative of the ethnic make-up of our locality during the study period. $47 \%$ of our local community identify as ethnicities other than White. In contrast, $90 \%$ of IPU admissions were White (2\% Asian/Asian British, 1\% Black/African/Caribbean/Black British and 7\% not recorded). Similarly, 91\% of people attending our DTU were White (2\% Asian/Asian British and $7 \%$ not recorded).

Conclusion The documented ethnicity of our service users does not reflect the ethnic diversity of our local populace. It is impossible to conclude from this project why ethnicities other than White are underrepresented. Potential barriers need to be investigated but might include a lack of awareness of the services we provide.

Since these data were collected, hospice staff have met with local religious leaders to demystify palliative care and establish if hospice services need to adapt to better serve our community.

\section{P-30 WHO IS HOSPICE MISSING?}

Giovanna Cruz, Diane Corrin, Cheryl Young, Lonan Oldam, Anne Mills, Sarah McGhee. Hospice Isle of Man, Douglas, Isle of Man

\subsection{6/bmjspcare-2019-HUKNC.54}

Background Hospice Isle of Man's aim is to provide access to those who could benefit regardless of diagnosis. The hospice began offering services to non-cancer patients in 2016. Little is known about how many could benefit or the extent of the hospice's involvement by disease category. The Isle of Man is not included in Hospice UK's PopNAT.

Aims To estimate the number of persons who could have potentially benefited from palliative and end-of-life care. To understand differences in hospice involvement by disease category.

Methods The Isle of Man Registry maintains weekly Excel sheets with death certificate data. We extracted variables of interest for the period between 1 January 2013 to 31 December 2018. We created a variable for palliative need based on cause of death where there was any mention of cancer, Alzheimer's/dementia, organ failure or a neurodegenerative condition. Using hospice records, we identified persons who received hospice care for the years 2016-2018. We estimated unmet need by comparing hospice involvement and presence of a condition likely to benefit from palliative care and compared hospice involvement by palliative disease category.

Results There were 5,005 deaths between 2013 and 2018, representing $1 \%$ of the population per year. Prior to 2016, the hospice cared for $30 \%$ of persons who died. By 2018, $39 \%$ had hospice care representing a $21 \%$ increase from 2016. Of all deaths between 2013 and 2018, 30\% had a palliative condition and hospice care. An additional 34\% had a palliative condition but no hospice care. The hospice was involved in $88 \%$ of all cancer cases but only in a quarter or less of cases with organ failure (28\%), neurodegenerative conditions (26\%), or Alzheimer's/dementia (16\%).

Conclusions Since 2016, more persons received hospice care. However, persons with non-cancer conditions who could benefit remained under-represented compared to those with cancer. Future studies are needed to understand the factors contributing to this difference. 